\title{
FBICM: Efficient Congestion Management for High-Performance Networks Using Distributed Deterministic Routing
}

\author{
Jesús Escudero-Sahuquillo ${ }^{1}$, Pedro García ${ }^{1}$, Francisco Quiles ${ }^{1}$, Jose Flich $^{2}$, \\ and Jose Duato ${ }^{2}$ \\ ${ }^{1}$ Dept. of Computing Systems, University of Castilla-La Mancha, Spain \\ \{jescudero,pgarcia, paco\}@dsi.uclm.es \\ 2 Dept. of Computer Engineering, Technical University of Valencia, Spain \\ \{jflich, jduato\}@gap.upv.es
}

\begin{abstract}
As the number of components in cluster-based systems increases, cost and power consumption also increase. One way to reduce both problems is using smaller networks with adequate congestion management mechanisms. Recent successful proposals (RECN) eliminate the negative effects of congestion, the Head-of-Line (HOL) blocking, leaving congestion harmless. RECN relies on source-based networks architectures, where the entire route is placed at packet headers before injection. Unfortunately, distributed table-based routing is also common in cluster-based networks, being InfiniBand the most prominent example. We propose a novel congestion management technique for distributed tablebased routing. The mechanism relies on additional congestion information located at routing tables. With this information HOL blocking is minimized by smartly using switch queues. Detailed memory organization and the way congestion information is updated/propagated is described. Preliminary results indicate that with modest resource requirements maximum network performance is kept regardless of congestion.
\end{abstract}

Keywords: High-Performance Interconnects, Congestion Control, Distributed Routing.

\section{Introduction}

During the last decades, the evolution of interconnection network technology has been really significant. Of course, this evolution has taken place in parallel with the proliferation of computing and communication systems based on these networks: Massive Parallel Processors, Local and System Area Networks, Clusters of PCs and Workstations, IP routers, and Networks-on-Chip. In order to work at maximum capacity, such systems demand low packets latencies and high network bandwidth. On the other hand, the popularity and utilization of these systems is constantly growing. As a clear example, more than half of the most powerful systems (top500 list) [1] are clusters. Consequently, there exists a great interest on achieving the best possible performance of the interconnection network and, in fact, researchers and designers have proposed many architectures and techniques with the aim of improving any key aspect of network functionality.

One of such aspects is the network behavior during congestion situations. These situations appear when several packet flows persistently request the same output inside a 
switch. In such situations, and assuming a lossless network (so, discarding packets is not allowed), packets from the involved flows arrive at the corresponding input buffers faster than they can cross to the requested output, thus these buffers finally collapse. Moreover, flow control propagates congestion throughout the network, following the reversal path of the flows contributing to congestion (thereby forming "congestion trees" across the network [2]). When congestion reaches many network points, the immediate consequence is a severe network performance degradation (throughput drops, latency increases exponentially).

The specific cause of this degradation is that congested flows may share some network resources (queues, links) with non-congested flows, thereby the former slowing the advance of the latter. In detail, in a queue storing packets belonging to congested and non-congested flows, a "congested packet" reaching the head of the queue will usually have to wait for a long period before being forwarded, and consequently all the other packets in the queue (both congested and non-congested) will suffer this delay. In general, this effect is known as Head-Of-Line (HOL) blocking, and it may limit the throughput of the switch up to about $58 \%$ of its peak value [3].

In modern high-speed interconnection networks, the use of some technique for solving the problems related to congestion has become actually mandatory, since current networks are usually designed using a low number of network components (due to the high cost and power consumption of such components), thereby decreasing the offered bandwidth and increasing congestion probability. In that sense, many proposed techniques can help to reduce the negative effects of congestion (see section 2), but none as satisfactorily as the technique known as Regional Explicit Congestion Notification (RECN) [456]. Contrary to common techniques that try to avoid or prevent congestion, RECN completely eliminates the HOL blocking produced by congested flows leaving congestion harmless. RECN requires a reduced set of additional resources per switch port, which are dynamically allocated for storing congested packets separately from non-congested ones. In this way, RECN keeps network performance at maximum during congestion situations at a moderate cost.

However, an essential requirement for any RECN implementation is the use of source-based routing where the entire path of a packet is encoded in its header. RECN identifies congested packets by comparing the explicit route stored in the header of each packet to explicit routes leading to congested points. Of course, this limitation prevents RECN from being applied in any network technology that uses table-based, distributed routing, like Infiniband [7]. Therefore, efficient congestion management on these technologies is still an open issue, while the use of such technologies is far from being unpopular.

In order to fill this significant technical gap, we present in this paper a new technique able to eliminate the HOL blocking produced by congested flows in networks implementing table-based, distributed deterministic routing. We have called this new technique Flow-Based Implicit Congestion Management (FBICM). FBICM eliminates HOL blocking following the same basic approach as RECN, so by detecting congested packets and separating them from non-congested ones. However, FBICM differs from RECN in many aspects, especially the following ones: 
1. Congested points are not addressed by means of explicit routes. Instead, FBICM identifies congested points implicitly, by keeping track of the different flows passing through these points.

2. Congested packets are not identified as packets following an explicit route to a congested point, but as packets belonging to flows involved in a congested situation.

3. Congestion information is associated to destinations whereas in RECN is associated to internal network points. Thus, totally new implementations are required.

As a consequence of these three basic differences, FBICM requires new formats for congestion notifications, and new policies for congestion detection, congestion information propagation and resource management. As we will show in this paper, we have carefully considered all these aspects in the design of FBICM, in such a way that FBICM finally offers the same performance as RECN, but in a different technological context. Summing up, FBICM offers the benefits of an efficient, cost-effective congestion management to interconnection networks that implement table-based, distributed deterministic routing.

The rest of the paper is organized as follows. Section 2 shows an overview of the existing related work. Next, in Section 3, the basics of the new FBICM mechanism are presented. In Section 4. FBICM is compared in terms of performance and resource needs to previously proposed techniques which also reduce or eliminate HOL blocking. Finally, in Section 5, some conclusions are drawn.

\section{Related Work}

The risk of congestion in interconnection networks is a well-known problem, and many strategies have been proposed. The simplest ones are, however, overdimensioning the network and/or dropping packets in congestion situations. However, none of them are suitable for modern interconnection networks due, respectively, to the high cost and consumption of current network components and to the lossless character of these networks.

Other more elaborated techniques have been specifically proposed for avoiding or eliminating congestion. For instance, proactive strategies are based on reserving network resources for each data transmission, requiring a traffic planification based on network status [8]. However, this status information is not always available, and the resource reservation procedure introduces significant overhead. On the other hand, reactive congestion management is based on notifying congestion to the sources contributing to its formation, in order to cease or reduce the traffic injection from those sources [9]. Unfortunately, these solutions may not be efficient due to the delay between congestion detection and notification.

Other strategies deal with the HOL blocking problem, thus, indirectly dealing with congestion. Several HOL blocking elimination strategies have been proposed: Virtual Output Queues (VOQs) [10], Dynamically Allocated Multiqueues (DAMQs) [11], congestion buffers [12], etc. Most of these techniques rely on allocating different buffers for storing separately packets belonging to different flows.

In general, traditional HOL blocking elimination techniques are feasible or effective, but not feasible and effective at the same time. For instance, the use of VOQs at network level requires as many queues at each port as end-points in the network, being so an 
effective but not scalable technique. A variation of VOQ uses as many queues at each port as output ports in a switch [13](eliminating switch-wide HOL blocking). So, this technique is feasible, but it does not eliminate completely network-wide HOL blocking.

In contrast to these techniques, RECN eliminates HOL blocking in an efficient and scalable manner. Like VOQs, RECN tries to separate congested and non-congested flows by storing them in different queues. Specifically, RECN adds a set of additional queues (set aside queues, SAQs) to the standard queues at every input and output port of a switch. While standard queues will store non-congested packets (RECN assumes that packets from non-congested flows can be mixed in the same buffer without producing significant HOL blocking), SAQs are dynamically allocated for storing packets passing through a specific congested point. SAQs are allocated only when congestion arises, and can be deallocated when congestion vanishes, so RECN uses these resources efficiently.

Every set of SAQs is controlled by means of a CAM (content addressable memory), in such a way that each CAM line contains information for managing an associated SAQ, including the information required for addressing a congested point. In that sense, RECN assumes the use of source deterministic routing, thereby addressing congested network points by means of explicit routes toward these points. These routes can be indicated by sets of "hops" stored in the CAM lines. Once a packet arrives at a port, the routes stored in the port CAM lines can be compared to the explicit route stored in the packet header, in order to know if the packet will cross any of the detected congested points. In this way, congested packets can be easily detected and stored in the corresponding SAQ, thereby preventing them from sharing standard queues with non-congested packets, and consequently avoiding HOL blocking.

Although the RECN basic mechanism described above has proved to be very efficient, it presents the obvious limitation of requiring the use of source deterministic routing. As we have already mentioned, the main aim of the new HOL blocking elimination technique presented in this paper (FBICM) is to solve this flaw by offering the same benefits as RECN, but in a table-based, distributed deterministic routing context. The following section describes how this goal can be achieved.

\section{FBICM Description}

In this section we describe FBICM and its operation. Firstly we focus on the assumed switch architecture: memory organization, routing and arbitration mechanisms, etc. Next, we explain in detail the main features of FBICM: congestion detection, queue allocation, etc., illustrating the descriptions by means of operation diagrams.

\subsection{Switch Architecture}

In Fig. 1, a scheme of the assumed switch architecture is shown. It is important to note that FBICM, just like RECN, does not limit the number of ports of a switch. We assume that both at input and output ports, Link Controllers (LC) coordinate the transfer of flow control units at each side of the physical channel. In that sense, a credit-based flow control mechanism at packet level has been assumed.

As can be seen in the scheme, there exist RAM memories only at input ports. This is because FBICM has been developed for Input Queued (IQ) switches, thus we assume 


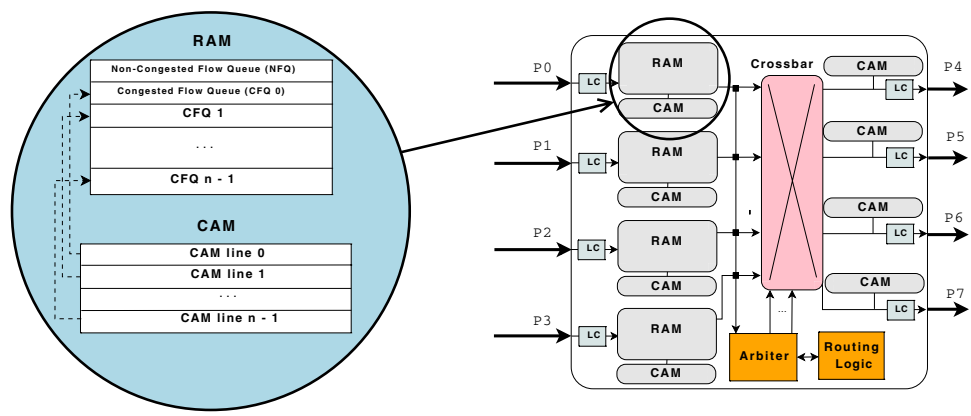

Fig. 1. Switch Architecture Assumed by FBICM (4x4 Switch)

that only switch input ports have queues. This kind of switches are very popular, and they are cheaper than Combined Input and Output Queued (CIOQ) switches, the former requiring less memory and resources for operating than the latter 1 . Input RAMs are organized in queues, dynamically managed. We assume two types of queues: noncongested flow queues (NFQs), where non-congested packets are stored, and congested flow queues (CFQs), where packets belonging to congested flows are placed. Moreover, associated with each set of CFQs, there is a content addressable memory (CAM), which contains information about the congested ports and the status of each CFQ. This port memory organization can be seen in the "zoom" include in Fig. 1

Although output ports have neither NFQs nor CFQs, FBICM requires a CAM per output port, in order to propagate congestion information from downstream input port CAMs to upstream input port CAMs, as we will explain later.

Regarding the routing mechanism, FBICM has been designed for networks using table-based, distributed deterministic routing, thus the Routing Logic is based on a routing table that indicates the output port for each incoming packet. We assume routing tables are filled during network setup according to some routing algorithm. In our experiments, we have used a self-routing algorithm for multistage networks, calculating the output port as a function of the destination of the packet. Therefore, the only routing information that packets need to include in their header is their destination (instead of a explicit, complete route).

On its side, the switch arbiter selects (following an "oldest-first" policy) routed packets for switching them through the crossbar, as long as the required input-output connection is possible (so, only if the corresponding crossbar internal path is free). The crossbar assumed for this model is a $\mathrm{NxN}$ multiplexed crossbar, where $\mathrm{N}$ is the number of input/output channels. Finally, the switching technique used for this model is Virtual Cut-Through (VCT).

\subsection{Congestion Detection}

A congestion situation happens when some packets request the same output port within the switch and this situation persists in time. In normal conditions the rate at which

\footnotetext{
${ }^{1}$ Note that the latest RECN version [6] was also designed for IQ switches.
} 


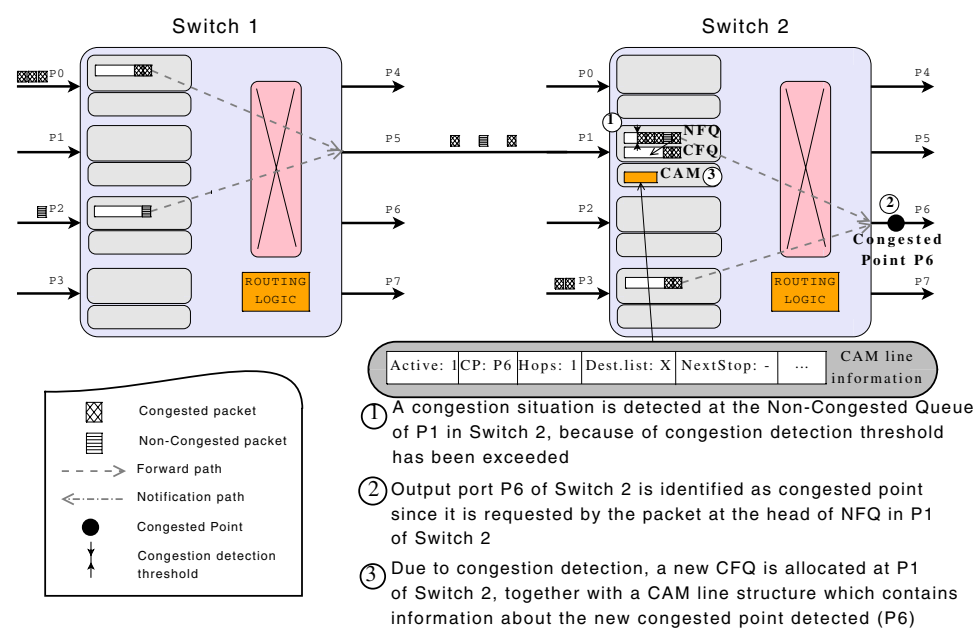

Fig. 2. Operation Example of Congestion Detection and Primary Queue Allocation in FBICM

packets arrive to a given input port will be approximately the same as packets leaving the output port, thus the occupation of non-congested flow queues should be low. In congestion situations, the NFQs at the switch input ports will fill, and it is necessary to detect these congestion situations as a first step for avoiding the HOL blocking that would appear.

FBICM detects congestion situations at input ports by enabling a detection threshold at non-congested flow queues. When the number of packets in a NFQ exceeds this threshold, a new congested point is identified. Specifically, we assume that is very likely that the packet which is allocated at the head of the NFQ belongs to a flow contributing to create congestion in the switch. Therefore, this detection method infers this packet is delaying the normal packet flow towards its requested output port and thus, this output port is probably a congested poin 2 , (so being the "root" of a growing congestion tree).

Actually, it is possible that the packet at the head of the NFQ is not a congested one. This would be, in fact a failure in the detection mechanism, but it will be corrected (as we will explain alter) by means of the packet post-processing and the deallocation policy of CFQs.

In Fig. 2 (pass 1), we can see a simple operation example about the situation described above. There are two types of packets: congested which are routed to P6 at switch 2, and non-congested which are routed to other destinations. The congested ones come from P0 in Switch 1 and P3 in Switch 2 and the non-congested ones come from $\mathrm{P} 2$ in Switch 1. Since packets from different input ports request the same output port, a contention situation appears and the normal flow of packets is delayed, causing a congestion situation. When the occupancy of the NFQ at input port P1 of switch 2 exceeds the detection threshold value (in this case the threshold value is five packets), the congestion detection takes place at this input port. Since the output port requested by the

\footnotetext{
${ }^{2}$ This detection method has been also used and tested in previous congestion management techniques [6], exhibiting a great accuracy.
} 


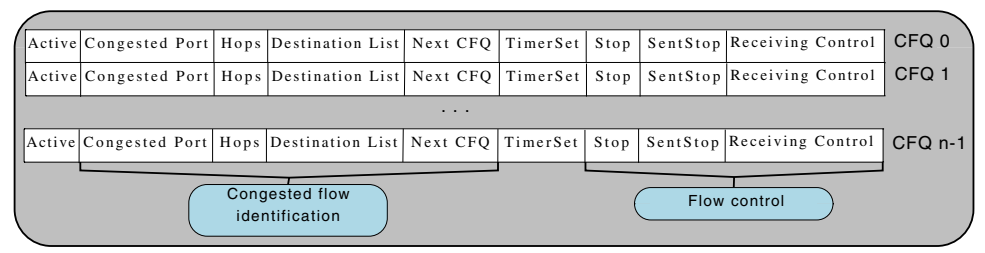

Fig. 3. CAM organization

packet at the head of the NFQ detecting congestion is P6, this output port is identified as a congested point (see Fig. 2 pass 2).

\subsection{Primary CFQ Allocation}

Once a switch output port is detected as a congested point, FBICM must separate incoming packets addressed to that congested point from incoming packets which do not request that port. In order to do that, a CFQ will be dynamically allocated at any input port that detects congestion, as well as an associated CAM line. The CAM line will contain the CFQ status information and also information for identifying the congested port.

Figure 3 shows in detail the organization of CAM. As can be seen, congestion information consists of four fields: Congested-Port, hops-to-reach, list-of-destinations, and NextCFQ. The Congested-Port field indicates an output port detected as congested from the input port, while the list-of-destinations field is intended to contain all the destinations that would lead incoming packets to request that congested port. In this way, given the destination of an incoming packet, it is possible to know immediately if it would request the congested port, thereby allowing to decide if it belongs to a congested flow 3 and it must be stored in the associated CFQ. Many destinations can be progressively added to the list-of-destinations, as congested packets with different destinations pass through the input port. The "hops-to-reach" field is used to measure (in hops) the distance from the CAM line input port to the congested point, thus it is only useful when congestion information is propagated upstream from the input port (see subsection 3.5). The "NextCFQ" field is used also during congestion information propagation.

On the other hand, the CFQ status information stored in each CAM line consists of four bits, indicating respectively if the CAM is active (Active bit), if the CAM is blocked by the CFQ-specific flow control (Stop bit), if it is receiving control information ("Receiving Control" bit), and if the congestion information has been propagated to the output ports of the upstream switch ("Sent Stop" bit).

In Fig. 2 (pass 3), we show a "primary" CFQ allocation example. As can be seen, a new CFQ is dynamically allocated after the congestion detection at port P1 of switch 2. The associated CAM line is filled in with information about congestion: congested port identifier (P6 in this case), number of hops to reach that port (1 in this case), and the destinations of packets that request the congested port from P1 (at this first moment, only one destination: $\mathrm{X}$ ).

\footnotetext{
${ }^{3}$ Note this is possible because we assume distributed deterministic routing; adaptive routing would not allow us to identify congested packets in that way.
} 
From this moment, once a new CFQ is allocated in the input port, FBICM will separate the congested flows by storing in that CFQ those incoming packets whose destination is in the list-of-destinations field of the corresponding CAM line, while incoming packets belonging to non-congested flows will be mapped to the NFQ. Note that congested packets are identified taking into account only its destination, thus source (explicit) routing is not required. Congested packet identification is performed by the Packet Processing mechanism.

List-of-Destinations Implementation. Take into account that the list-of-destinations field described above is just a simplification (necessary for an straightforward understanding of FBICM basics), but it should be implemented efficiently in real systems, as it could become the bottleneck of the new logic or even consume many resources. One effective way to implement such list per CAM line is to add new fields to the routing table. Assuming a routing table with $N$ entries (one per destination), a switch with $p$ ports, and a FBICM mechanism with $c$ CFQs per port, each entry for a destination node $d$ would contain the following fields:

1. Output port (OP). This is the output port provided to the arbitration logic for the packet being routed, computed from packet destination.

2. CAMline at the output port (CLOP). A $c$-bit register indicating the CAM lines at the output port (OP) where the destination $d$ is mapped into. Notice that the destination may be mapped on more than one CAM line, thus we need one bit per CAM line (c).

3. CAM line at the input port (CLIP). A vector with $p$ elements, each with $c$ bits. Each vector element represents the mappings of the destination $d$ into the CAM lines on a particular port. Thus, each element contains $c$ bits, one per CAM line.

4. Notification Sent bit (NSB). One bit per input port ( $p$ ). Each bit will be set on each time a notification is sent upstream for the $d$ destination through a given port.

As can be seen, several new fields are required at the routing table. Assuming a 1024-node system implemented with 8-port switches, a routing table with no congestion management would require $3 \mathrm{~KB}$ memory space (1024 destinations, each one coding a 3-bit output port). With the addition of the congestion management mechanism with 8 CFQs per port, the memory requirements for the routing table increase to $83 \mathrm{~KB}$ (1024 destinations, 3-bit for OP field, 4 bits for CLOP field, 32 bits for CLIP field, 8 bits for NSB field 4 .

\subsection{Packet Processing}

The post-processing mechanism decides if a packet belongs to a congested flow, thereby also deciding if it must be stored in a CFQ or in the NFQ. This mechanism looks around the packets arriving at the head of all the input port queues (NFQ and CFQs), and compares the packet destination to the CAM lines information (specifically, to the

\footnotetext{
${ }^{4}$ Although such memory increase may be acceptable, as future work we plan to reduce the memory requirements for FBICM. We plan to address caching mechanisms as destinations potentially will reach a switch through a small subset of ports.
} 


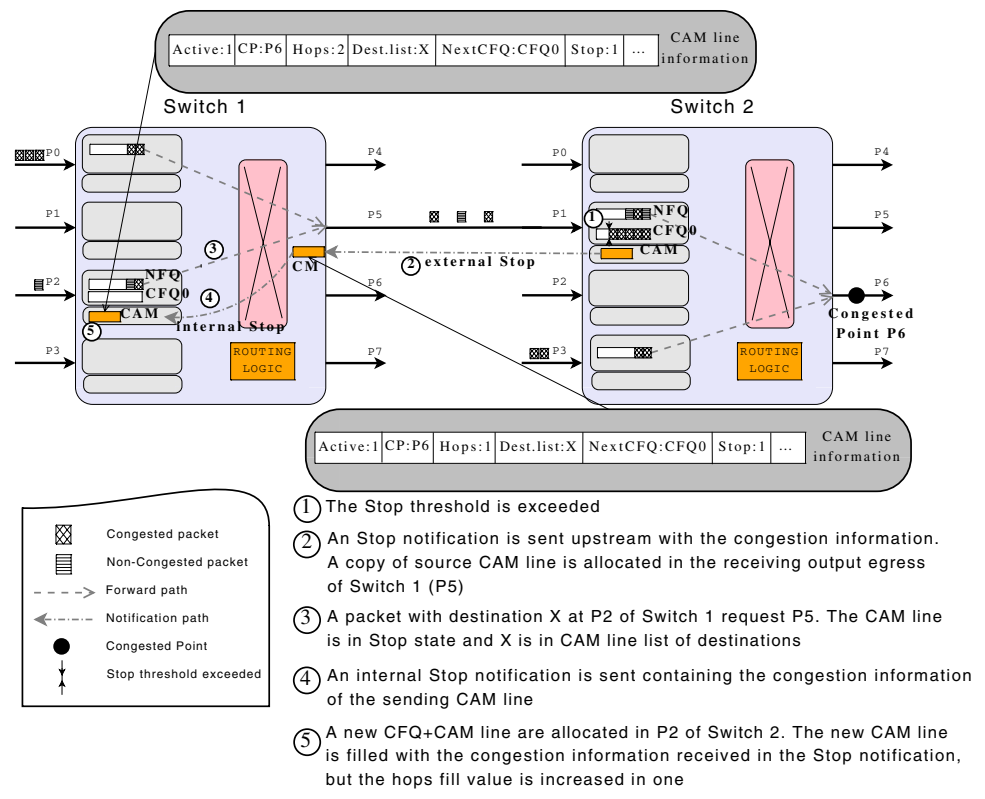

Fig. 4. Operation Example of Congestion Information Propagation

destinations included in the list-of-destinations field). If the packet is at the head of the NFQ and the comparison result is a match, the packet will be considered as congested and will be moved to the corresponding CFQ in the same input port. Otherwise, the packet will remain in the NFQ and the post-processing mechanism will set it as ready for the switch arbiter. Note that this mechanism leaves at the head of the NFQ only non-congested packets, thus HOL blocking is avoided at the NFQ. On the other hand, once a packet stored in a CFQ reaches the head of a that queue, it will be set as ready for the arbiter. Note that this post-processing mechanism assures the in-order delivery of packets because, although congested and non-congested flows are separated, it keeps the order of packets belonging to the same flow (either congested or non-congested).

Additionally, the post-processing mechanism selects which NFQ or CFQ can forward a packet at each moment, by means of a round robin policy among all the queues (NFQ and active CFQs) at each input port.

\subsection{Congestion Information Propagation}

In the same way that FBICM sets a congestion detection threshold in the NFQ, a threshold (Stop threshold) is used, when congestion persists for long, both for avoiding CFQ overflow and for propagating congestion information. An example of this is shown in Fig. 4.

Basically, when the occupancy of a CFQ in an input port exceeds the Stop threshold, an Stop notification including the information of the associated CAM line is sent to the upstream output port. When an output port receives an external Stop notification, 
it checks if there is already an active CAM line containing the same received information. If not, it will allocate a new CAM line, filling it with that information (so, active output CAM lines will be exact copies of downstream CAM lines). Otherwise, if there were already an allocated CAM for the received information, the Stop notification is considered just as a flow control message. In both cases (new CAM line allocation or not), the involved CAM line will be set to Stop state, and as a consequence this output port will not accept packets belonging to packets flows whose destinations are included in the list-of-destinations field of that CAM line. Note that this means that the output port may control the flow of congested packets without requiring explicit routes for identifying them.

On the other hand, when the occupancy of an input port CFQ that sent an Stop notification decreases enough (until a given threshold), a Go notification is sent upstream, with the opposite effect than Stop ones. Thus, upon reception of a Go notification, an output port CAM line will turn off the Stop bit, entering the Go state and so unblocking the flow of packets.

Inside of the switch, "internal" Stop notifications may be sent when some input port request to forward a packet to an output port with active CAM lines. If one of these CAM lines is in Stop state and the packet destination is in its list-of-destinations field, an internal Stop notification, containing all the CAM line information, will effectively be sent to the input port. In this way, congestion information from downstream input port CAM lines are transmitted through output port CAM lines to all the input ports crossed by the flows contributing to congestion. Note again that specific routes are not necessary for identifying congested packets.

On its side, an input port receiving an internal Stop notification may allocate or not a new CFQ + CAM line, depending on the existence at that port of an active CAM line containing the same congestion information received. In the case of a new allocation, the CAM line will be filled with the received information, but increasing the "hops-toreach" value by one (this reflects the growing distance to the "root of the congestion tree"). Otherwise, the internal Stop notification will be considered just as a flow control message. Again, in both cases (new allocation or just flow control), the involved CAM line will be set to Stop state. Of course, internal Go notifications are also sent for unblocking packet flow.

The newly allocated input port CFQ will store congested packets (again, identified only from their destinations), and may repeat the propagation process, spreading upstream the congestion information. In this way, when a congestion situation persists FBICM will identify the congested flows at any network point, and packets belonging to them will be stored in the corresponding CFQs. Thus, congested flows will not interact with the non-congested ones, thereby avoiding HOL blocking.

\section{FBICM Evaluation}

In this Section we will evaluate FBICM by comparing it to other HOL blocking elimination techniques, specifically to the RECN version for IQ switches (RECN-IQ) [6], Virtual Output Queues at network level (VOQNet) and Virtual Output Queues at switch level (VOQSw). 
We will use different scenarios of traffic load and network size. For this purpose we have used an ad-hoc event-driven simulator developed in $\mathrm{C}$ language that models the network at the register transfer level. Firstly, we will describe the modeling assumptions and the main parameters used in the simulations. Secondly, we will analyze the obtained results.

\subsection{Simulated Scenarios}

The simulator models Bidirectional Multistage Interconnection Networks (BMINs) with switches, endnodes, and links, allowing different connectivity patterns (e.g.perfect shuffle, butterfly,...) and network sizes. For this evaluation, we have used two netwok configurations:

1. Configuration 1: 64 hosts connected with a $64 \times 64$ perfect shuffle BMIN. This network includes 48 switches in three stages, each switch having 8 bidirectional ports.

2. Configuration 2: 256 hosts connected with a perfect shuffle $256 \times 256$ BMIN. This network includes 256 switches in four stages, each switch having 8 bidirectional ports.

In all the experiments, a BMIN self-routing deterministic algorithm has been used. For the RECN-IQ experiments, source routing has been modeled, thus this algorithm has been used to generate explicit routes included in packet headers. For FBICM, VOQNet and VOQSw, table-based, distributed routing has been modeled, thus in these cases the routing algorithm has been used for filling the routing tables.

At switches, packets are forwarded from input queues to output queues through a multiplexed crossbar, modeled with a speedup of 1 (link bandwidth is equal to crossbar bandwidth). RAM memories have been modeled at each input port of the switch, with different sizes depending on the simulated HOL blocking elimination technique. For FBICM, RECN-IQ and VOQSw, we have used 8 KB RAMs, but VOQNet requires larger memories since the number of queues per port is higher in this case. In particular, VOQNet requires $16 \mathrm{~KB}$ memories for $64 \times 64 \mathrm{MINs}$ and $64 \mathrm{~KB}$ memories for $256 \times 256$ MINs. In the case of FBICM, a maximum of 8 active CFQs per port have been allowed, and also 8 active SAQs the case of RECN-IQ.

In all the experiments, endnodes (hosts) are modeled as connected to switches using Input Adapters (IAs). Every IA is modeled with a fixed number of $N$ admittance queues (where $N$ is the total number of endnodes), and a variable number of injection queues, which follow a scheme similar to that of the output ports of a switch. When a message is generated, it is stored in the admittance queue assigned to its destination, and is packetized before being transferred to an injection queue. We have used 64-byte packets.

Of course, in order to compare the results of the considered HOL blocking elimination techniques, the simulator also allows experiments to be performed using either FBICM, RECN-IQ, VOQNet or VOQSw.

Regarding traffic load, the simulator allows to use either synthetic traffic or traces, and we have used both in our experiments. The considered synthetic traffic patterns are shown in table 1 Note that in some patterns (\#1, \#2 and \#5) traffic follows a completely uniform (random) destination distribution, while in others (\#3, \#4 and \#6), a percentage 
Table 1. Synthetic traffic patterns used in the evaluation

\begin{tabular}{|c|c|c|c|c|c|c|c|c|c|}
\hline & & \multicolumn{3}{|c|}{ Random Traffic } & \multicolumn{5}{c|}{ Hot-Spot Traffic } \\
\hline $\begin{array}{c}\text { Traffic } \\
\text { case }\end{array}$ & $\begin{array}{c}\text { Network } \\
\text { (BMIN) }\end{array}$ & \# Srcs & Dest & $\begin{array}{c}\text { Generation } \\
\text { rate }\end{array}$ & \# Srcs & Dest & $\begin{array}{c}\text { Generation } \\
\text { rate }\end{array}$ & $\begin{array}{c}\text { Start } \\
\text { time }\end{array}$ & $\begin{array}{c}\text { End } \\
\text { time }\end{array}$ \\
\hline$\# 1$ & $64 \times 64$ & $100 \%$ & random & $100 \%$ & $0 \%$ & - & - & - & - \\
$\# 2$ & $64 \times 64$ & $100 \%$ & random & incremental & $0 \%$ & - & - & - & - \\
$\# 3$ & $64 \times 64$ & $75 \%$ & random & $100 \%$ & $25 \%$ & 32 & $100 \%$ & $1000 \mu \mathrm{s}$ & $1300 \mu \mathrm{s}$ \\
$\# 4$ & $64 \times 64$ & $75 \%$ & random & incremental & $25 \%$ & 32 & incremental & $0 \mu \mathrm{s}$ & $3000 \mu \mathrm{s}$ \\
\hline$\# 5$ & $256 \times 256$ & $100 \%$ & random & $100 \%$ & $0 \%$ & - & - & - & - \\
$\# 6$ & $256 \times 256$ & $75 \%$ & random & $100 \%$ & $25 \%$ & 123 & $100 \%$ & $1000 \mu \mathrm{s}$ & $1300 \mu \mathrm{s}$ \\
\hline
\end{tabular}

of sources generate traffic addressed to a unique, hot-spot destination, thereby creating congestion. In all these patterns, packet generation rate is indicated as a relative percentage of link bandwidth.

Regarding traces, the ones used in our experiments were provided by HewlettPackard Labs, and they include all the I/O activity generated from 1/14/1999 to $2 / 28 / 1999$ at the disk interface of the cello system. As these traces are almost nine years old, we have applied a time compression factor to the traces.

In all the experiments, the simulator offers different metrics. For our evaluation, we have considered network throughput (as a function of time or traffic load) as the main metric for measuring the performance of the networks when the different HOL blocking elimination techniques are used. Additionally, we have also considered FBICM consumption of CFQs (the total amount of active CFQs in the network at each moment) and RECN-IQ consumption of SAQs, in order to compare resource requirements of both techniques.

\subsection{Evaluation Results}

Figures 5 (a) and 5 (b) show network throughput results for completely uniform synthetic traffic patterns in a $64 \times 64$ BMIN. As we can see, FBICM obtains the same throughput as VOQNet and RECN-IQ, and it improves slightly the results of VOQSw. Due to the uniform traffic properties, the congestion situations are very short and occur in many different points in the network, thus none of the techniques faces great difficulties for keeping network performance at a good level.

However, for hot-spot scenarios leading to congestion situations in the same network, results are significantly different, as can be seen in Fig.6 (a) and 6 (b). In these cases, network throughput significantly decreases when VOQSw are used, while FBICM, RECNIQ and VOQNet achieve better performance, keeping network throughput at maximum even during congestion. Note, however, that VOQNet achieves these results by using far more queues per input port (specifically, 64 queues) than FBICM (1 queue + a maximum of 8 queues) or RECN-IQ (also 1 queue + a maximum of 8 queues). Since results obtained by these three techniques are quite similar, we can conclude that both FBICM and RECN-IQ eliminate HOL blocking more efficiently than VOQNet. But note also that FBICM achieves these results for distributed deterministic routing interconnects, while RECN-IQ cannot be implemented in such technologies.

On the other hand, Figs. 5(c) and 6(c) compare the total consumption of CFQs (FBICM) and SAQs (RECN-IQ) along simulation time when traffic cases \#1 and \#3 are used. As can be seen, results are very similar, thus we can conclude that FBICM 


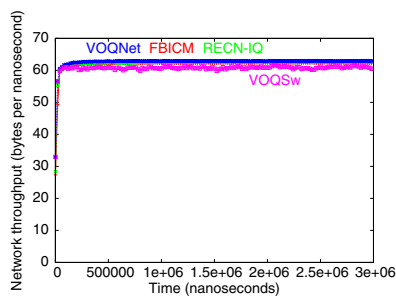

(a) Throughput vs. Time

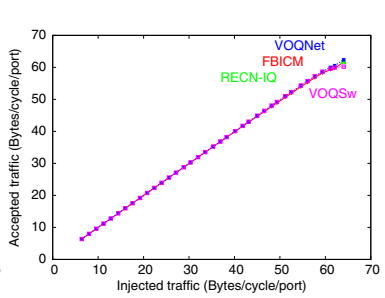

(b) Throughput vs. Load

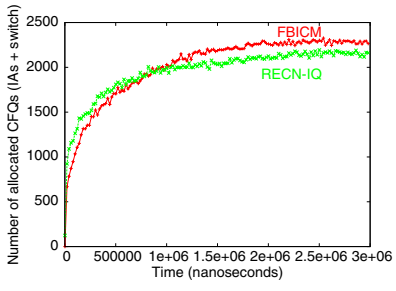

(c) Total CFQ Consumption vs. Time

Fig. 5. Network Throughput vs. Time and Load, and Total CFQ Consumption for Configuration 1 (Traffic Cases \#1 and \#2)

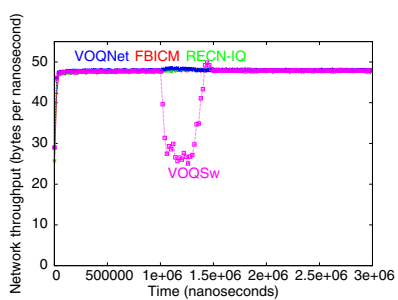

(a) Throughput vs. Time

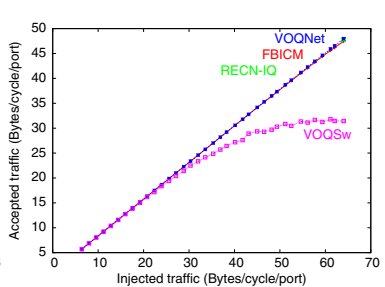

(b) Throughput vs. Load

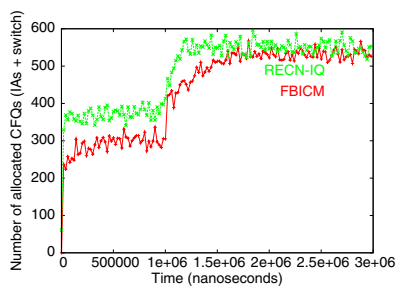

(c) Total CFQ Consumption vs. Time

Fig. 6. Network Throughput vs. Time and Load, and Total CFQ Consumption for Configuration 1 (Traffic Cases \#3 and \#4)

require approximately the same amount of resources for allocating congested flows than RECN-IQ, thereby the former being approximately as efficient as the latter.

Figure 7 shows throughput results for a larger network (specifically, a $256 \times 256$ BMIN) when either completely uniform traffic (Fig. 7(a)) or hot-spot traffic (Fig. 7(b)) are present. In the uniform traffic case, FBICM achieves the same results as RECNIQ with the same (and moderate) resources, while VOQNet achieves slightly better results. Note, however, that in this case VOQNet uses $64 \mathrm{~KB}$ memories per port for implementing 256 queues per port, while $8 \mathrm{~KB}$ per port are used by FBICM. As the congestion situations are very short in this traffic pattern, VOQSw obtains almost the same results as the other techniques. However, in the hot-spot case, the behavior of VOQSw is very poor, while we can see that FBICM achieved throughput is the same than VOQNet (but again the former using fewer queues than the latter). From this results we can conclude that also for large networks, FBICM keeps network performance at maximum even during congestion situations, thereby being a scalable technique.

In the last figure of the evaluation (Fig. 8) it can be seen the similar throughput achieved by VOQNet, RECN-IQ and FBICM, when traces are used as traffic load in a $64 \times 64$ BMIN. It can be observed that, regardless of the traces compression factor, FBICM achieves the maximum throughput also for real traffic.

Summing up, all the evaluation results show that maximum performance is achieved by FBICM regardless of network size or traffic pattern, even in congestion situations. VOQNet obtains similar results than FBICM, but requires far more resources, especially 


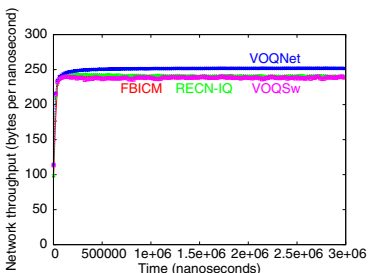

(a) Throughput vs. Time (Traffic Case \#5)

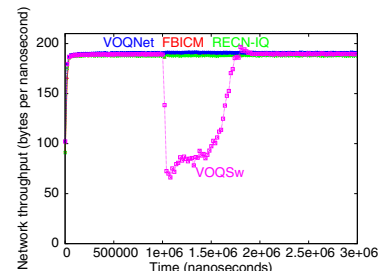

(b) Throughput vs. Time (Traffic Case \#6)

Fig. 7. Network Throughput vs. Time for Configuration 2

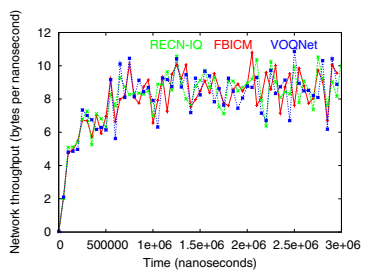

(a) Compression Factor Set to 20

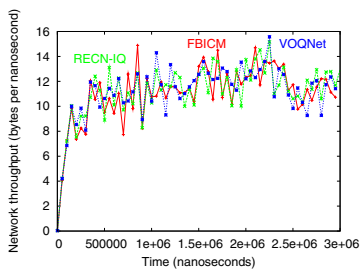

(b) Compression Factor Set to 40

Fig. 8. Network Throughput for Traces in a 64x64 BMIN

in large networks. VOQSw consumes similar resources than FBICM, but its performance is poor in hot-spot traffic situations, regardless of network size. On the other hand, results for RECN-IQ are similar to the FBICM ones, but in a different interconnect context.

\section{Conclusions}

Congestion situations are a serious menace for the performance of current interconnection networks. In these situations, packet flows contributing to congestion slow the advance of other flows due to the HOL blocking effect, thereby degrading overall network performance. Many techniques have been proposed for solving this problem, the one known as RECN achieving the best performance by eliminating HOL blocking in a truly efficient and scalable way. Since RECN requires the use of deterministic source routing, networks using table-based, distributed routing cannot benefit from a RECN implementation.

In this paper we have proposed FBICM, a new HOL blocking elimination technique for network technologies that implement table-based, distributed deterministic routing. Especially, we have described how implicit information about congested points can be stored and propagated (once congestion is detected) without requiring explicit routes, and how packets belonging to congested flows can be identified taking into account just their destination.

We have also shown evaluation results that demonstrate that FBICM achieves for distributed routing networks the same performance reached by the latest RECN version 
(RECN-IQ) for source routing networks, since both techniques keep network performance at maximum even in serious congestion situations, regardless network size. Results also show that FBICM is more efficient than VOQnet (because the latter requires far more resources) and VOQSw (that exhibits a poor performance in congestion situations). Taking all this into account, we can conclude that FBICM offers efficient and scalable HOL blocking elimination to networks based on distributed deterministic routing networks.

\section{Acknowledgments}

This work is jointly supported by the following projects: Consolider Ingenio-2010CSD2006-00046, TIN2006-15516-C04-02, PCC08-0078 (with PhD. grant A08/078).

\section{References}

1. Top-500-List: Web page (July 2008), http: / / www . top500 . org

2. García, P.J., Flich, J., Duato, J., Johnson, I., Quiles, F.J., Naven, F.: Dynamic evolution of congestion trees: Analysis and impact on switch architecture. In: Conte, T., Navarro, N., Hwu, W.-m.W., Valero, M., Ungerer, T. (eds.) HiPEAC 2005. LNCS, vol. 3793, pp. 266285. Springer, Heidelberg (2005)

3. Karol, M.J., Hluchyj, M.G., Morgan, S.P.: Input versus output queueing on a space-division packet switch. IEEE Trans. on Commun. COM-35, 1347-1356 (1987)

4. Duato, J., Johnson, I., Flich, J., Naven, F., García, P.J., Nachiondo, T.: A new scalable and cost-effective congestion management strategy for lossless multistage interconnection networks. In: Proceedings of the 11th Symposium on High Performance Computer Architecture (HPCA) (2005)

5. García, P.J., Flich, J., Duato, J., Johnson, I., Quiles, F.J., Naven, F.: Efficient, scalable congestion management for interconnection networks. IEEE Micro 26(5), 52-66 (2006)

6. Mora, G., García, P.J., Flich, J., Duato, J.: RECN-IQ: A cost-effective input-queued switch architecture with congestion management. In: Proceedings of 36th International Conference on Parallel Processing (ICPP 2007) (2007)

7. InfiniBand Trade Association: InfiniBand architecture specification volume 1. Release 1.0 (October 2000)

8. Wang, M., Siegel, H.J., Nichols, M.A., Abraham, S.: Using a multipath network for reducing the effects of hot spots. IEEE Transactions on Parallel and Distributed Systems 6(3), 252-268 (1995)

9. Thottetodi, M., Lebeck, A., Mukherjee, S.: Self-tuned congestion control for multiprocessor networks. In: Proc. of 7th. Int. Symp. on High Performance Computer Architecture (February 2001)

10. Dally, W., Carvey, P., Dennison, L.: Architecture of the Avici terabit switch/router. In: Proceedings of the 6th Symposium on Hot Interconnects, pp. 41-50 (1998)

11. Tamir, Y., Frazier, G.: Dynamically-allocated multi-queue buffers for vlsi communication switches. IEEE Transactions on Computers 41(6) (June 1992)

12. Smai, A., Thorelli, L.: Global reactive congestion control in multicomputer networks. In: Proc. 5th Int. Conference on High Performance Computing (1998)

13. Anderson, T., Owicki, S., Saxe, J., Thacker, C.: High-speed switch scheduling for local-area networks. ACM Transactions on Computer Systems 11(4), 319-352 (1993) 